\title{
Strained 1-Azabicyclo[1.1.0]butanes in the synthesis of Azetidinethiocarboxylate derivatives
}

\author{
Woznicka, M ; Urbaniak, K ; Mloston, G ; Heimgartner, H
}

\begin{abstract}
The reaction of 3-phenyl-1-azabicyclo[1.1.0]butane (1a) with chlorodithio-formates (5) at room temperature yielded 3-chloro-3-phenylazetidine-1-carbodithioates (6). The same products were obtained in a two-step procedure by treatment of 1a with thiophosgene to give azetidine-1-carbothioyl chloride (7a), followed by treatment with the corresponding sulfane. 3-Chloro-3-phenylazetidine-1-thiocarbamides (8) and the corresponding O-methyl 1-carbothioates (9) were prepared by the reaction of compounds (7) with amines and methanol, respectively. These reactions open a new access to derivatives of azetidine-1carboxylic acid.
\end{abstract}

Posted at the Zurich Open Repository and Archive, University of Zurich

ZORA URL: https://doi.org/10.5167/uzh-51265

Journal Article

Accepted Version

Originally published at:

Woznicka, M; Urbaniak, K; Mloston, G; Heimgartner, H (2006). Strained 1-Azabicyclo[1.1.0]butanes in the synthesis of Azetidinethiocarboxylate derivatives. Heterocycles, 69:351-364. 
HETEROCYCLES, Vol. 69, 2006, pp. 351 - 364. (C) The Japan Institute of Heterocyclic Chemistry

Received, 20th July, 2006, Accepted, 21st September, 2006, Published online, 26th September, 2006. COM-06-S(O)43

STRAINED 1-AZABICYCLO[1.1.0]BUTANES IN THE SYNTHESIS OF AZETIDINETHIOCARBOXYLATE DERIVATIVES

\title{
Marta Woznicka, ${ }^{\mathrm{a}, 1}$ Katarzyna Urbaniak, ${ }^{\mathrm{a}}$ Grzegorz Mloston, ${ }^{\mathrm{a} *}$ and Heinz Heimgartner $^{\mathrm{b} *}$
}

a: Department of Organic and Applied Chemistry, University of Lodz, Narutowicza 68, PL-90-136 Lodz, Poland; E-mail: gmloston@uni.lodz.pl

b: Institute of Organic Chemistry, University of Zurich, Winterthurerstrasse 190, CH-8057 Zürich, Switzerland; E-mail: heimgart@oci.unizh.ch

\begin{abstract}
The reaction of 3-phenyl-1-azabicyclo[1.1.0]butane (1a) with chlorodithio-formates (5) at room temperature yielded 3-chloro-3phenylazetidine-1-carbodithioates (6). The same products were obtained in a twostep procedure by treatment of 1a with thiophosgene to give azetidine-1carbothioyl chloride (7a), followed by treatment with the corresponding sulfane. 3-Chloro-3-phenylazetidine-1-thiocarbamides (8) and the corresponding $O$-methyl 1-carbothioates (9) were prepared by the reaction of compounds (7) with amines and methanol, respectively. These reactions open a new access to derivatives of azetidine-1-carboxylic acid.
\end{abstract}

\section{INTRODUCTION}

The smallest bicyclic systems containing one $\mathrm{N}$-atom are 1- and 2-azabicyclo[1.1.0]butanes. Whereas the latter were postulated as unstable intermediates only, several representatives of the former have been described $^{2,3}$ (and refs. cited therein). ${ }^{4}$ In the last two decades, numerous reactions were reported, in which 1-azabicyclo[1.1.0]butanes were explored as versatile reagents. ${ }^{2,6}$ The most important reaction is the addition of electrophilic agents of type R-X (e.g. ( $\mathrm{RCO})_{2} \mathrm{O}, \mathrm{ClCO}_{2} \mathrm{R}, \mathrm{N}_{3} \mathrm{CO}_{2} \mathrm{R}, \mathrm{TsCl}, \mathrm{TsN} \mathrm{N}_{3}, \mathrm{HF}$, etc) across the weakest $\mathrm{C}-\mathrm{N}$ bond to give azetidine derivatives. As an example, the addition of benzyl 
azidoformate with 3-phenyl-1-azabicyclo[1.1.0]butane (1a) leading to 1-benzyloxyazetidine (2) is shown in Scheme 1. ${ }^{7}$

\section{Scheme 1}<smiles>c1ccccc1</smiles>

$1 \mathrm{a}$

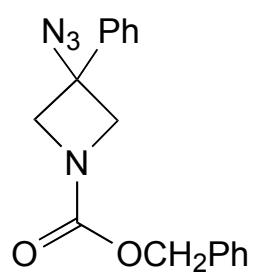

2

Reactions of thiophosgene with primary or secondary amines are applied in the synthesis of thiocarbamoyl chlorides, which are useful intermediates for the preparation of thiocarbamates, dithiocarbamates and thioureas. ${ }^{8-11}$ An alternative way to prepare dithiocarbamates is the reaction of amines with chlorodithioformates. ${ }^{12-14}$ Cyclic secondary amines such as pyrrolidine and morpholine are known to react easily with thiophosgene to give either the corresponding thiocarbamoyl chlorides or thioureas. ${ }^{15,16}$ However, in the case of the parent aziridine, the intermediate (3) undergoes a ring opening accompanied by elimination of $\mathrm{HCl}$ to give 2-chloroethyl isothiocyanate (4, Scheme 2). ${ }^{17}$ The corresponding reaction with azetidine has not been reported. As thiocarbamoyl derivatives are of general interest with respect to their biological activity, we decided to elaborate a synthesis of such azetidine derivatives starting with 1-azabicyclo[1.1.0]butanes (1).

\section{Scheme 2}

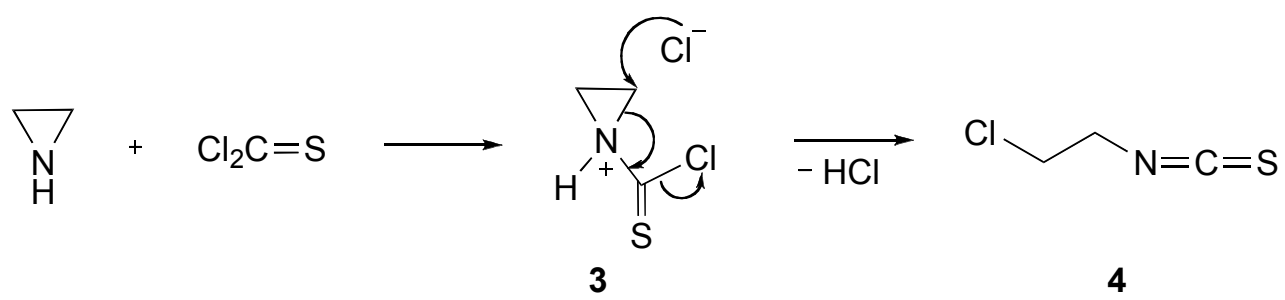

\section{RESULTS AND DISCUSSION}

The reaction of 1a with phenyl chlorodithioformate (5a) was carried out at room temperature in $\mathrm{CH}_{2} \mathrm{Cl}_{2}$. After $1 \mathrm{~h}$ (TLC control), the starting materials were consumed, the solvent was evaporated, and the product (6a) was isolated as a crystalline material by means of prep. TLC. In the ${ }^{1} \mathrm{H}-\mathrm{NMR}$ spectrum, the 
signals of the two $\mathrm{CH}_{2}$ groups appear as a complex multiplet (4.87-5.00 ppm); the corresponding ${ }^{13} \mathrm{C}$ absorptions are located at 69.4 and $70.3 \mathrm{ppm}$. These data indicate a hindered rotation within the thiocarbamoyl moiety. ${ }^{18}$ The hindered rotation about the $\mathrm{C}-\mathrm{N}$ bond in N,N-disubstituted thioamides is well documented. ${ }^{19}$ On the other hand, symmetrically N,N-disubstituted dithiocarbamates have been reported to show only one signal for two equivalent atoms of the two N-substituents in the ${ }^{1} \mathrm{H}-\mathrm{NMR}$ spectrum. $^{20}$ Only recently, the NMR spectra of such compounds registered at 200 and $400 \mathrm{MHz}$ evidenced small differences in chemical shifts of equivalent atoms in the ${ }^{1} \mathrm{H}$ - as well as the ${ }^{13} \mathrm{C}-\mathrm{NMR}$ spectrum. ${ }^{21}$ Both, MS and elemental analyses confirmed the formation of a 1:1-adduct of $\mathbf{1 a}$ and $\mathbf{5 a}$, and finally, the molecular structure of $\mathbf{6 a}$ was established by X-Ray crystallography (Scheme 3, Figure). The analogous reactions of $\mathbf{1 a}$ with benzyl and propyl chlorodithioformate (5b and $\mathbf{5 c}$ ), yielded the azetidine derivatives (6b) and (6c), respectively (Scheme 3).

\section{Scheme 3}

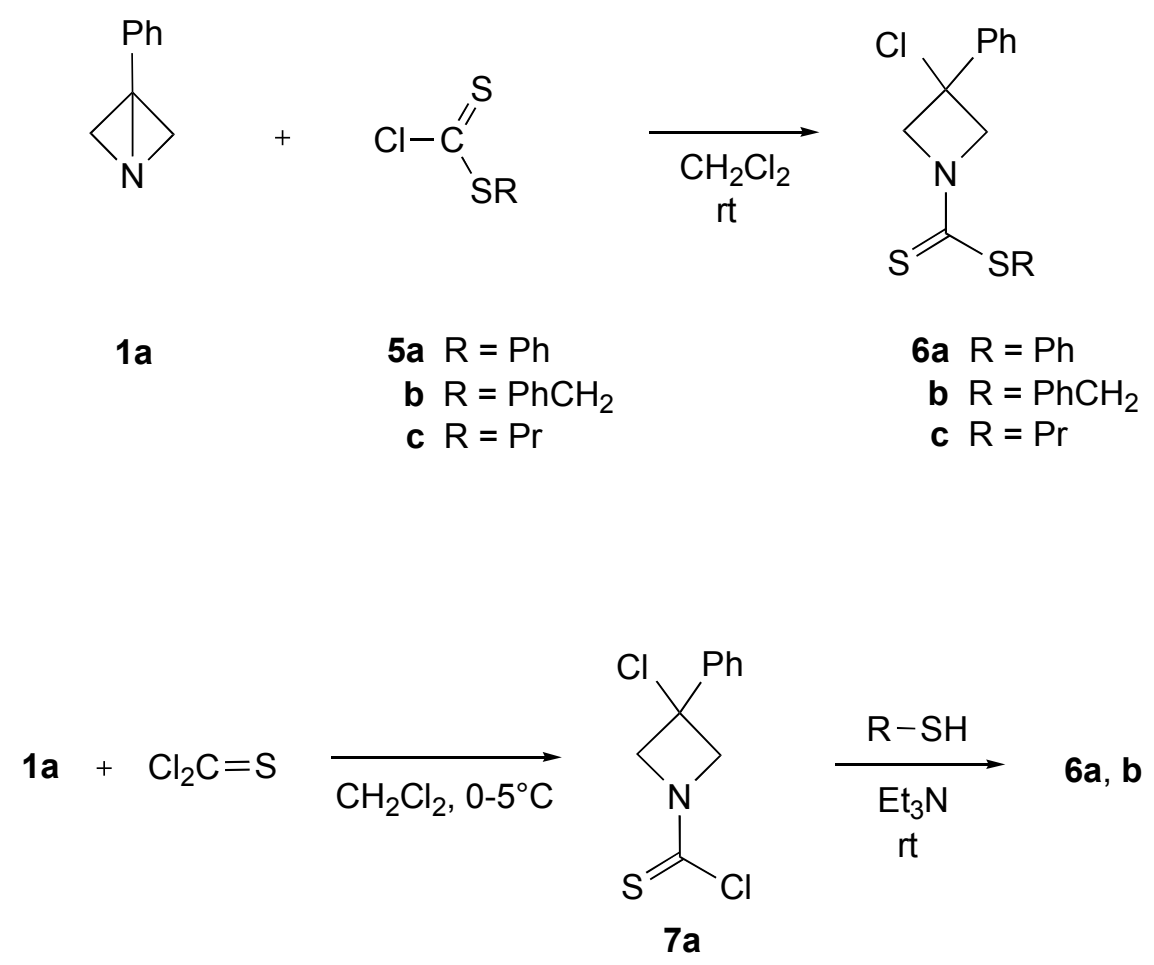

The dithiocarbamates (6a) and (6b) have also been prepared by an alternative method using thiocarbamoyl chloride (7a), which was easily accessible by addition of thiophosgene and 1a (Scheme 3). The reaction of thiophosgene with $\mathbf{1 a}$ is exothermic, and the mixture had to be cooled. In the ${ }^{1} \mathrm{H}-\mathrm{NMR}$ spectrum of 7a, the $\mathrm{CH}_{2}$ groups appear as a multiplet at 4.78-5.28 ppm. The ${ }^{13} \mathrm{C}-\mathrm{NMR}$ spectrum shows a characteristic absorption for $\mathrm{C}=\mathrm{S}$ at $172.6 \mathrm{ppm}\left(N, N\right.$-dimethylthiocarbamoyl chloride: $187.3 \mathrm{ppm}^{21 \mathrm{~b}}$ or $\left.173.1 \mathrm{ppm}^{23}\right)$ as well as two triplets for $\mathrm{CH}_{2}$ groups $(70.0$ and $71.3 \mathrm{ppm})$ and one singlet for the 
quaternary C-atom (58.4 ppm) of the azetidine moiety. Without isolation, the solution of the crude 7a was treated with two equivalents of benzenethiol and benzyl sulfane, respectively, in the presence of one equivalent of $\mathrm{Et}_{3} \mathrm{~N}$. After $24 \mathrm{~h}$ and aqueous workup, $6 \mathbf{a}$ and $\mathbf{6 b}$ were isolated. The attempted reaction of 7a with tert-butyl sulfane failed, and after $24 \mathrm{~h}$ the ${ }^{1} \mathrm{H}-\mathrm{NMR}$ spectrum revealed the presence of unconsumed $7 \mathbf{a}$.

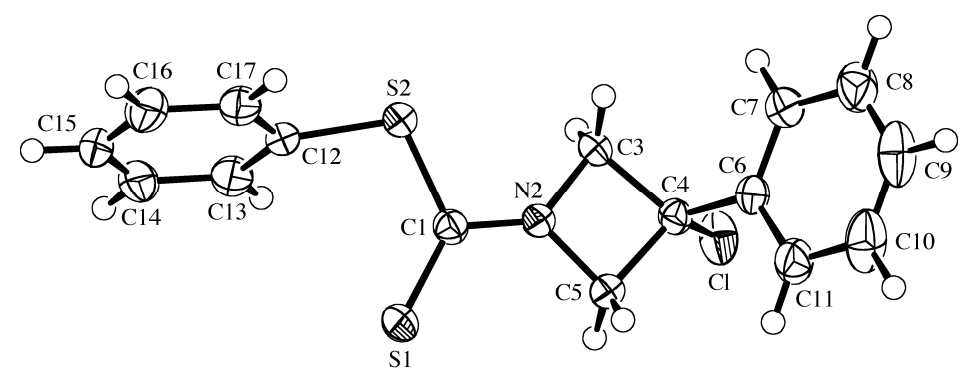

Figure 1. ORTEP plot $^{22}$ of the molecular structure of 6 a $(50 \%$ probability ellipsoids; arbitrary numbering of atoms).

The in situ prepared $7 \mathbf{a}$ was also treated with piperidine or morpholine ( 2 equiv.) to give, after $1 \mathrm{~h}$ at room temperature, the expected thioureas $(\mathbf{8 a})$ and $(\mathbf{8 b})$, respectively (Scheme 4). Similarly, the reaction with aniline led to 8c. In contrast to dithiocarbamates of type 6, the ${ }^{13} \mathrm{C}-\mathrm{NMR}$ spectra of thioureas (8) showed only one $\mathrm{CH}_{2}$-absorption for the azetidine ring.

\section{Scheme 4}

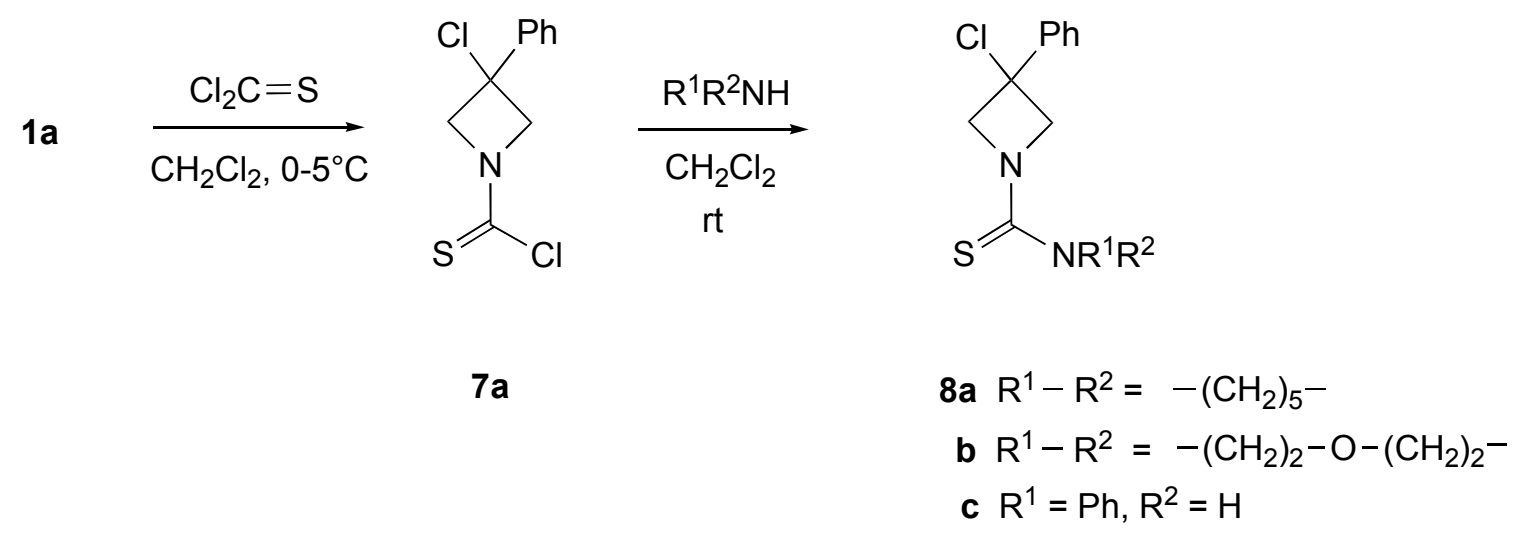

Furthermore, the $\alpha$ - and $\beta-\mathrm{C}$ atoms of the piperidine and morpholine residues showed one signal for two $\mathrm{CH}_{2}$ groups in each case. This phenomenon indicates that the rotation barrier for the $\mathrm{CN}$ bonds in the thiourea derivatives (8) is significantly lower than in the dithiocarbamates (6). This observation fits well with the reported low rotational barrier in tetrasubstituted thioureas. ${ }^{24}$ 
With the aim of preparing symmetrical thioureas bearing two azetidine rings, the reaction of 1a with thiophosgene in the ratio of 2:1 was carried out. After addition of thiophosgene to the solution of 1a in $\mathrm{CH}_{2} \mathrm{Cl}_{2}$ at $0-5^{\circ} \mathrm{C}$ and stirring of the mixture for $10 \mathrm{~min}$, the solvent was evaporated, and a viscous oily residue was obtained, which was identified as $7 \mathbf{a}$. None of the expected thiourea could be detected.

In extension of the reactions of $7 \mathbf{a}$ with thiols and amines, $\mathrm{MeOH}$ was used as an $O$-nucleophile. The crude 7a, prepared in a typical manner (1:1 ratio of $\mathbf{1 a}$ and $\left.\mathrm{Cl}_{2} \mathrm{C}=\mathrm{S}\right)$, was dissolved in $\mathrm{MeOH}$, and the solution was left at room temperature over night. A crystalline product was isolated and identified as thiocarbamate (9a) (Scheme 5). The $\mathrm{MeO}$ group of this product absorbs at $4.00\left({ }^{1} \mathrm{H}\right)$ and $58.0 \mathrm{ppm}\left({ }^{13} \mathrm{C}\right)$. In the ${ }^{13} \mathrm{C}-\mathrm{NMR}$ spectrum, the signal of the $\mathrm{C}=\mathrm{S}$ group appears at $189.4 \mathrm{ppm}$, and two signals for the two $\mathrm{CH}_{2}$ groups were found at 67.3 and $68.7 \mathrm{ppm}$. The quarternary azetidine $\mathrm{C}$-atom absorbs at $61.0 \mathrm{ppm}$. In the case of 2,2-dimethyl-3-phenyl-1-azabicyclo[1.1.0]butane (1b), the crude 1:1-adduct (7b) was obtained in almost quantitative yield. Its ${ }^{13} \mathrm{C}$-NMR spectrum shows two $\mathrm{C}=\mathrm{S}$ signals at 174.7 and $173.1 \mathrm{ppm}$ and two sets of two Me signals at 25.6/23.5 and 25.2/22.5 ppm, which indicates the presence of two rotamers in almost equal amounts. The reaction of this product with $\mathrm{MeOH}$ afforded $\mathbf{9 b}$ as a crystalline material. On the basis of the ${ }^{1} \mathrm{H}-\mathrm{NMR}$ spectrum (signals at $1.83 / 1.08$ and $2.03 / 1.32 \mathrm{ppm}$ ), the ratio of the two rotamers was determined to $c a .5: 1$.

\section{Scheme 5}

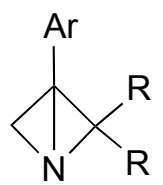

$$
\frac{\mathrm{Cl}_{2} \mathrm{C}=\mathrm{S}}{\mathrm{CH}_{2} \mathrm{Cl}_{2}, 0-5^{\circ} \mathrm{C}}
$$<smiles>[R]C1([R])N(C(=S)Cl)CC1(Cl)Br</smiles>

$$
\text { 1a } \begin{aligned}
\mathrm{Ar} & =\mathrm{Ph}, \mathrm{R}=\mathrm{H} \\
\text { b } \mathrm{Ar} & =\mathrm{Ph}, \mathrm{R}=\mathrm{Me} \\
\text { c } \mathrm{Ar} & =4-\mathrm{FC}_{6} \mathrm{H}_{4}, \mathrm{R}=\mathrm{Me}
\end{aligned}
$$

$$
\begin{aligned}
\text { 7a } \mathrm{Ar} & =\mathrm{Ph}, \mathrm{R}=\mathrm{H} \\
\text { b } \mathrm{Ar} & =\mathrm{Ph}, \mathrm{R}=\mathrm{Me} \\
\text { c } \mathrm{Ar} & =4-\mathrm{FC}_{6} \mathrm{H}_{4}, \mathrm{R}=\mathrm{Me}
\end{aligned}
$$

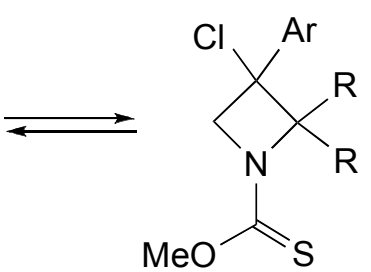

$$
\begin{aligned}
\text { 9a } \mathrm{Ar} & =\mathrm{Ph}, \mathrm{R}=\mathrm{H} \\
\text { b } \mathrm{Ar} & =\mathrm{Ph}, \mathrm{R}=\mathrm{Me} \\
\text { c } \mathrm{Ar} & =4-\mathrm{FC}_{6} \mathrm{H}_{4}, \mathrm{R}=\mathrm{Me}
\end{aligned}
$$

In summary, the results described in this paper show that the reactions of 1-azabicyclo[1.1.0]butanes (1) with chlorodithioformates and thiophosgene, respectively, open straightforward access to the hitherto unknown thiocarbamoyl derivatives of azetidine. In contrast to the reaction of $\mathrm{Cl}_{2} \mathrm{C}=\mathrm{S}$ with the structurally related aziridine, which after ring opening and elimination of $\mathrm{HCl}$ leads to a chlorinated isothiocyanate (see Scheme 2), compounds (1) undergo conversion to the less strained and relatively stable adducts (7). In spite of this difference, the reaction mechanisms of these two transformations follow a similar pathway, typical for three-membered nitrogen heterocycles (Scheme 6). 
Scheme 6
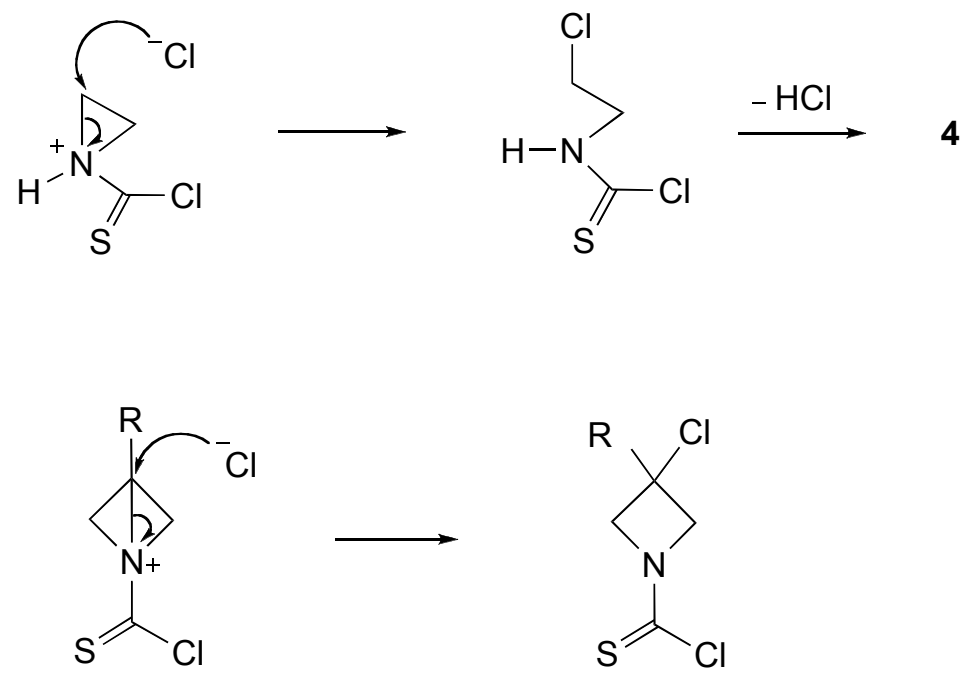

7

\section{EXPERIMENTAL}

General remarks. Melting points ( $\mathrm{mp}$ ) were determined in capillary using a Meltemp 2 apparatus and are uncorrected. IR spectra (KBr pellets or neat) were recorded with a Nexus spectrophotometer. ${ }^{1} \mathrm{H}$ - and ${ }^{13} \mathrm{C}$ NMR spectra were registered with a Tesla BS 687 instrument (80 MHz and $20 \mathrm{MHz}$, respectively) or a Bruker 300 (300 MHz and $75 \mathrm{MHz}$, respectively) spectrometer using TMS $(\delta=0 \mathrm{ppm})$ as an internal standard. MS (CI) were recorded on a Finnigan-Mat-90 or Finnigan-SSQ-700 spectrometer. Elemental analyses were performed in the Analytical Laboratory of the University of Zurich or in the Laboratory of the Polish Academy of Sciences (CBMiM) in Lodz.

Starting materials. 3-Phenyl-1-azabicyclo[1.1.0]butane (1a), 2,2-dimethyl-3-phenyl-1azabicyclo[1.1.0]butane (1b), and 2,2-dimethyl-3-(4-fluorophenyl)-1-azabicyclo[1.1.0]butane (1c) were prepared according to a known protocol from trimethylsulfonium iodide, butyllithium and the corresponding azirine. ${ }^{25}$ Phenyl chlorodithioformate (5a), benzyl chlorodithioformate (5b), and propyl chlorodithioformate (5c) were synthesized from the corresponding sulfane and thiophosgene in $\mathrm{CHCl}_{3} / \mathrm{aq}$. $\mathrm{NaOH}(\mathbf{5 a})^{26}$ or in $\mathrm{CS}_{2}(\mathbf{5 b}, \mathbf{5 c}){ }^{27}$

2,2-Dimethyl-3-(4-fluorophenyl)-1-azabicyclo[1.1.0]butane (1c). Yield: $3.20 \mathrm{~g}$ (65\%). Colorless, thick oil distilled in a Kugelrohr at $80^{\circ} \mathrm{C} / 0.2$ Torr. IR (KBr): $1522 s, 1223 s, 833 s, 607 m, 553 s .{ }^{1} \mathrm{H}-\mathrm{NMR}\left(\mathrm{CDCl}_{3}\right)$ : 1.14, $1.18(2 s, 2 \mathrm{Me}), 2.49,2.62\left(A B, J=1.6 \mathrm{~Hz}, \mathrm{CH}_{2} \mathrm{~N}\right), 6.90-7.46(m, 4$ arom. $\mathrm{H}) .{ }^{13} \mathrm{C}-\mathrm{NMR}\left(\mathrm{CDCl}_{3}\right)$ : 12.8, $22.8(2 \mathrm{Me}), 41.3\left(\mathrm{Me}_{2} C\right), 54.2\left(\mathrm{CH}_{2} \mathrm{~N}\right), 68.4\left(\mathrm{C}_{\mathrm{q}}\right), 115.3\left(d,{ }^{2} J_{\mathrm{C}, \mathrm{F}}=21.7 \mathrm{~Hz}, 2\right.$ arom. $\left.\mathrm{CH}\right), 130.2(d$, 
${ }^{3} J_{\mathrm{C}, \mathrm{F}}=8.3 \mathrm{~Hz}, 2$ arom. CH), $130.6\left(d,{ }^{4} J_{\mathrm{C}, \mathrm{F}}=2.9 \mathrm{~Hz}\right.$, arom. $\left.\mathrm{C}_{\mathrm{q}}\right), 162.5\left(d,{ }^{1} J_{\mathrm{C}, \mathrm{F}}=246.4 \mathrm{~Hz}\right.$, arom. $\left.\mathrm{C}_{\mathrm{q}} \mathrm{F}\right)$. CI-MS: $179(12), 178\left(100,[M+1]^{+}\right)$.

Reaction of 1a with chlorodithioformates (5). General procedure A (GPA). A mixture of 1 mmol of 1a and $1 \mathrm{mmol}$ of the corresponding chlorodithioformate in $1 \mathrm{~mL}$ of $\mathrm{CH}_{2} \mathrm{Cl}_{2}$ was stirred magnetically for ca. $1 \mathrm{~h}$. Then, the solvent was evaporated and the product was isolated after preparative layer chromatography using plates precoated with silica and hexane $/ \mathrm{CH}_{2} \mathrm{Cl}_{2} 3: 2$ as the eluent. Analytically pure samples were obtained by crystallization from $\mathrm{MeOH} / \mathrm{CH}_{2} \mathrm{Cl}_{2}, \mathrm{Et}_{2} \mathrm{O}$ and hexane, respectively.

Reaction of 7a with thiophenol and benzyl sulfane. General procedure B (GPB). A solution of the crude $7 \mathbf{a}$ in $2 \mathrm{~mL}$ of $\mathrm{CH}_{2} \mathrm{Cl}_{2}$ was treated with a mixture of $101 \mathrm{mg}(1 \mathrm{mmol})$ of $^{2} \mathrm{Et}_{3} \mathrm{~N}$ and $2 \mathrm{mmol}$ of the corresponding thiol in $1 \mathrm{~mL}$ of $\mathrm{CH}_{2} \mathrm{Cl}_{2}$ at $\mathrm{rt}$. The mixture was stirred for $24 \mathrm{~h}$, the solution was diluted with $7 \mathrm{~mL}$ of $\mathrm{CH}_{2} \mathrm{Cl}_{2}$ and washed first with a $2 \%$ aqueous solution of $\mathrm{NaOH}$, then with a $2 \%$ aqueous solution of $\mathrm{HCl}$, and finally with water. The organic phase was separated and dried over $\mathrm{MgSO}_{4}$. Analytically pure samples were obtained after crystallization from a mixture of $\mathrm{MeOH}$ and $\mathrm{CH}_{2} \mathrm{Cl}_{2}$ (reaction with thiophenol) or after preparative layer chromatography using plates precoated with silica and hexane $/ \mathrm{CH}_{2} \mathrm{Cl}_{2}$ 1:1 as the eluent and subsequent crystallization from diethyl ether.

Phenyl 3-chloro-3-phenylazetidine-1-carbodithioate (6a). Yield: $160 \mathrm{mg}$ (50\%; GPA) and $130 \mathrm{mg}$ (41\%; GPB). Colorless crystals; mp $142-145^{\circ} \mathrm{C}\left(\mathrm{MeOH} / \mathrm{CH}_{2} \mathrm{Cl}_{2}\right)$. IR (KBr): $1467 s, 1438 s, 1176 s, 982 m, 748 m$, $699 m .{ }^{1} \mathrm{H}-\mathrm{NMR}\left(\mathrm{CDCl}_{3}\right): 4.87-5.00\left(m, 2 \mathrm{CH}_{2} \mathrm{~N}\right), 7.38-7.52\left(m, 10\right.$ arom. H). ${ }^{13} \mathrm{C}-\mathrm{NMR}\left(\mathrm{CDCl}_{3}\right): 60.9$ $\left(\mathrm{C}_{\mathrm{q}}\right), 69.4,70.3\left(2 \mathrm{CH}_{2} \mathrm{~N}\right), 125.6,128.8,129.0,129.2,130.2$, 136.5 (10 arom. $\left.\mathrm{CH}\right), 129.5,140.6$ (2 arom. $\left.\mathrm{C}_{\mathrm{q}}\right), 195.4(\mathrm{C}=\mathrm{S})$. CI-MS: $323(7), 322(38), 321\left(17,[M+1]^{+}\right), 320\left(100, M^{+}\right)$. Anal. Calcd for $\mathrm{C}_{16} \mathrm{H}_{14} \mathrm{NClS}_{2}$ : C, 60.08; H, 4.41; N, 4.38; S, 20.05. Found: C, 60.03; H, 4.44; N, 4.36; S, 19.83 .

Benzyl 3-chloro-3-phenylazetidine-1-carbodithioate (6b). Yield: $240 \mathrm{mg}$ (72\%; GPA) and $120 \mathrm{mg}(36 \%$; GPB). Colorless crystals; mp 52-54 ${ }^{\circ} \mathrm{C}\left(\mathrm{Et}_{2} \mathrm{O}\right)$. IR (KBr): 1479s, 1440s, 1171s, 979s, 725m, 695s, $614 m$. ${ }^{1} \mathrm{H}-\mathrm{NMR}\left(\mathrm{CDCl}_{3}\right): 4.56\left(s, \mathrm{CH}_{2} \mathrm{~S}\right), 4.76,4.82\left(A B, J=11.1 \mathrm{~Hz}, \mathrm{CH}_{2} \mathrm{~N}\right), 4.88,4.97(A B, J=12.1 \mathrm{~Hz}$, $\left.\mathrm{CH}_{2} \mathrm{~N}\right), 7.25-7.42$ (m, 10 arom. H). ${ }^{13} \mathrm{C}-\mathrm{NMR}\left(\mathrm{CDCl}_{3}\right): 40.7\left(\mathrm{CH}_{2} \mathrm{~S}\right), 61.1\left(\mathrm{C}_{\mathrm{q}}\right), 68.8,70.2\left(2 \mathrm{CH}_{2} \mathrm{~N}\right)$, 125.5, 127.5, 128.6, 128.8, 128.9, 129.1 (10 arom. $\mathrm{CH}), 136.1,140.6$ (2 arom. $\left.\mathrm{C}_{\mathrm{q}}\right), 195.2(\mathrm{C}=\mathrm{S}) . \mathrm{CI}-\mathrm{MS}$ : 336 (42), $334\left(100, M^{+}\right), 300$ (26), 178 (23). Anal. Calcd for $\mathrm{C}_{17} \mathrm{H}_{16} \mathrm{NClS}_{2}$ : C, 61.15; H, 4.83; N, 4.19; S, 19.2. Found: C, 60.16; H, 4.85; N, 4.02; S, 18.30 .

Propyl 3-chloro-3-phenylazetidine-1-carbodithioate (6c). Yield: $220 \mathrm{mg}$ (77\%; GPA). Colorless crystals; mp 46-48 ${ }^{\circ} \mathrm{C}$ (hexane). IR (KBr): 1485vs, 1448s, 1436s, 1422m, 1172vs, 977s, 718m, 693s, 619m, $524 m$. 
${ }^{1} \mathrm{H}-\mathrm{NMR}\left(\mathrm{CDCl}_{3}\right): 1.02\left(t, J=7.4 \mathrm{~Hz}, \mathrm{MeCH}_{2}\right), 1.67-1.79\left(\mathrm{~m}, \mathrm{MeCH}_{2} \mathrm{CH}_{2}\right), 3.26\left(t, J=7.5 \mathrm{~Hz}, \mathrm{CH}_{2} \mathrm{~S}\right)$, 4.76-4.97 (m, $\left.2 \mathrm{CH}_{2} \mathrm{~N}\right), 7.33-7.43(m, 10$ arom. $\mathrm{H}) .{ }^{13} \mathrm{C}-\mathrm{NMR}\left(\mathrm{CDCl}_{3}\right): 13.3\left(\mathrm{MeCH}_{2}\right), 22.4$ $\left(\mathrm{MeCH}_{2} \mathrm{CH}_{2} \mathrm{~S}\right), 38.0\left(\mathrm{MeCH}_{2} \mathrm{CH}_{2} \mathrm{~S}\right), 61.1\left(\mathrm{C}_{\mathrm{q}}\right), 68.8,70.0\left(2 \mathrm{CH}_{2} \mathrm{~N}\right), 125.6,128.7,128.9$ (5 arom. $\left.\mathrm{CH}\right)$, 140.7 (arom. $\mathrm{C}_{\mathrm{q}}$ ), $196.2(\mathrm{C}=\mathrm{S})$. CI-MS: 288 (40), $287\left(16,[M+1]^{+}\right), 286\left(100, M^{+}\right), 252$ (28). Anal. Calcd for $\mathrm{C}_{13} \mathrm{H}_{16} \mathrm{NClS}_{2}$ : C, 54.62; H, 5.64; N, 4.90; S, 22.44. Found: C, 54.67, H, 5.69, N, 4.85, S, 22.24.

Reaction of azabicyclobutanes (1) with thiophosgene. General procedure. A solution of 1 mmol of the corresponding azabicyclobutane (1) in $1 \mathrm{~mL}$ of $\mathrm{CH}_{2} \mathrm{Cl}_{2}$ in an ice-water bath was stirred magnetically and $115 \mathrm{mg}(1 \mathrm{mmol})$ of thiophosgene in $1 \mathrm{~mL}$ of $\mathrm{CH}_{2} \mathrm{Cl}_{2}$ was added. The stirring was continued for 5 min. After evaporation of the solvent, the crude 7 was analyzed without purification.

3-Chloro-3-phenylazetidine-1-carbothioyl chloride (7a). Yield: $241 \mathrm{mg}$ (98\%). Yellowish, thick oil. IR (neat): $1513 m, 1500 m, 1460 m, 1447 m, 1173 m, 990 m .{ }^{1} \mathrm{H}-\mathrm{NMR}\left(\mathrm{CDCl}_{3}\right): 4.78-4.98\left(m, 2 \mathrm{CH}_{2} \mathrm{~N}\right), 7.35-$ 7.47 (m, 5 arom. H). ${ }^{13} \mathrm{C}-\mathrm{NMR}\left(\mathrm{CDCl}_{3}\right): 58.4\left(\mathrm{C}_{\mathrm{q}}\right), 70.0,71.3\left(2 \mathrm{CH}_{2} \mathrm{~N}\right), 125.6,129.1,129.1$ (5 arom. $\mathrm{CH}), 140.0$ ( arom. $\mathrm{C}_{\mathrm{q}}$ ), $172.6(\mathrm{C}=\mathrm{S}) . \mathrm{CI}-\mathrm{MS}: 250$ (11), $248(69), 247\left(12,[M+1]^{+}\right), 246\left(100, M^{+}\right), 212$ (25), $210\left(45,[M-\mathrm{Cl}]^{+}\right)$. Anal. Calcd for $\mathrm{C}_{10} \mathrm{H}_{9} \mathrm{NCl}_{2} \mathrm{~S}: \mathrm{C}, 48.79 ; \mathrm{H}, 3.69 ; \mathrm{N}, 5.69$. Found: C, 48.52; H, $3.71 ; \mathrm{N}, 5.64$.

3-Chloro-2,2-dimethyl-3-phenylazetidine-1-carbothioyl chloride (7b). Yield: $266 \mathrm{mg}$ (97\%). Yellowish, thick oil. IR (neat): $1488 \mathrm{br}, 1443 \mathrm{~m}, 1128 \mathrm{~m}, 738 \mathrm{~m}, 697 \mathrm{~m}, 645 \mathrm{~m}, 586 \mathrm{~m} .{ }^{1} \mathrm{H}-\mathrm{NMR}\left(\mathrm{CDCl}_{3}\right)$ : major rotamer: 1.26, $1.99(2 s, 2 \mathrm{Me}), 4.53,5.06\left(A B, J=12.8 \mathrm{~Hz}, 2 \mathrm{CH}_{2} \mathrm{~N}\right), 7.21-7.57$ ( $m, 5$ arom. $\left.\mathrm{H}\right)$; minor rotamer: 1.35, $2.05(2 s, 2 \mathrm{Me}), 4.65,5.20\left(A B, J=12.8,2 \mathrm{CH}_{2} \mathrm{~N}\right), 7.21-7.57(m, 5$ arom. $\mathrm{H}) .{ }^{13} \mathrm{C}-\mathrm{NMR}\left(\mathrm{CDCl}_{3}\right)$ : major rotamer: 23.5, $25.6(2 \mathrm{Me}), 66.1\left(2 \mathrm{CH}_{2} \mathrm{~N}\right), 72.1\left(\mathrm{Me}_{2} C\right), 84.3\left(\mathrm{C}_{\mathrm{q}}\right), 129.6,131.6(5$ arom. $\mathrm{CH})$, $140.8\left(\operatorname{arom} . \mathrm{C}_{\mathrm{q}}\right), 173.1(\mathrm{C}=\mathrm{S})$; minor rotamer: 22.5, $25.2(2 \mathrm{Me}), 68.0\left(2 \mathrm{CH}_{2} \mathrm{~N}\right), 71.9\left(\mathrm{Me}_{2} \mathrm{C}\right), 83.0\left(\mathrm{C}_{\mathrm{q}}\right)$, 129.5, 131.7 (5 arom. CH), 140.4 (arom. $\mathrm{C}_{\mathrm{q}}$ ), 174.8 (C=S). CI-MS: 278 (14), 277 (13), 276 (68), 275 (21, $\left.[M+1]^{+}\right), 274\left(100, M^{+}\right), 240(25), 238\left(60,[M-\mathrm{Cl}]^{+}\right), 196(18)$. Anal. Calcd for $\mathrm{C}_{12} \mathrm{H}_{13} \mathrm{NCl}_{2} \mathrm{~S}$ : C, 52.56; H, 4.78; N, 5.11; S, 11.69. Found: C, 52.37; H, 4.97; N, 4.98; S, 11.63.

3-Chloro-2,2-dimethyl-3-(4-fluorophenyl)azetidine-1-carbothioyl chloride (7c). Yield: $278 \mathrm{mg}$ (95\%). Yellowish, thick oil. IR (KBr): 1490br, 1443s, 1236s, 1156m, 1123m, 842m, 829m, 818m, 754m, 591m, $569 m .{ }^{1} \mathrm{H}-\mathrm{NMR}\left(\mathrm{CDCl}_{3}\right)$ : major rotamer: 1.25, $1.98(2 \mathrm{~s}, 2 \mathrm{Me}), 4.53,5.02\left(A B, J=12.8 \mathrm{~Hz}, \mathrm{CH}_{2} \mathrm{~N}\right)$, 6.96-7.48 ( $m, 5$ arom. H); minor rotamer: 1.34, $2.04(2 s, 2 \mathrm{Me}), 4.66,5.16\left(A B, J=12.8 \mathrm{~Hz}, \mathrm{CH}_{2} \mathrm{~N}\right)$, 6.96-7.48 (m, 5 arom. H). ${ }^{13} \mathrm{C}-\mathrm{NMR}\left(\mathrm{CDCl}_{3}\right)$ : major rotamer: 23.5, $25.6(2 \mathrm{Me}), 66.3\left(2 \mathrm{CH}_{2} \mathrm{~N}\right), 71.3$ $\left(\mathrm{Me}_{2} C\right), 84.3\left(\mathrm{C}_{\mathrm{q}}\right), 118.3\left(d,{ }^{2} J_{\mathrm{C}, \mathrm{F}}=22.6 \mathrm{~Hz}, 2\right.$ arom. $\left.\mathrm{CH}\right), 131.7\left(d,{ }^{3} J_{\mathrm{C}, \mathrm{F}}=8.8 \mathrm{~Hz}, 2\right.$ arom. $\left.\mathrm{CH}\right), 136.9(d$, 
${ }^{4} J_{\mathrm{C}, \mathrm{F}}=3.6 \mathrm{~Hz}$, arom. $\left.\mathrm{C}_{\mathrm{q}}\right), 166.2\left(d,{ }^{1} J_{\mathrm{C}, \mathrm{F}}=253.2 \mathrm{~Hz}\right.$, arom. $\left.\mathrm{C}_{\mathrm{q}} \mathrm{F}\right), 174.8(\mathrm{C}=\mathrm{S})$; minor rotamer: $22.5,25.3$ $(2 \mathrm{Me}), 68.2\left(2 \mathrm{CH}_{2} \mathrm{~N}\right), 71.5\left(\mathrm{Me}_{2} C\right), 83.0\left(\mathrm{C}_{\mathrm{q}}\right), 118.3\left(d,{ }^{2} J_{\mathrm{C}, \mathrm{F}}=22.6 \mathrm{~Hz}, 2\right.$ arom. $\left.\mathrm{CH}\right), 131.6\left(d,{ }^{3} J_{\mathrm{C}, \mathrm{F}}=\right.$ $8.8 \mathrm{~Hz}, 2$ arom. $\mathrm{CH}), 136.9\left(d,{ }^{4} J_{\mathrm{C}, \mathrm{F}}=3.6 \mathrm{~Hz}\right.$, arom. $\left.\mathrm{C}_{\mathrm{q}}\right), 166.2\left(d,{ }^{1} J_{\mathrm{C}, \mathrm{F}}=253.2 \mathrm{~Hz}\right.$, arom. $\left.\mathrm{C}_{\mathrm{q}} \mathrm{F}\right), 173.2$ $(\mathrm{C}=\mathrm{S}) . \mathrm{CI}-\mathrm{MS}: 294(68), 292\left(100, M^{+}\right), 258(26), 256\left(67,[M-\mathrm{Cl}]^{+}\right)$.

Reaction of 7a with piperidine, morpholine, and aniline. General procedure. To a solution of $1 \mathrm{mmol}$ of the crude 7a dissolved in $2 \mathrm{~mL}$ of $\mathrm{CH}_{2} \mathrm{Cl}_{2}$ was treated with $2 \mathrm{mmol}$ of the corresponding amine in 1 $\mathrm{mL}$ of $\mathrm{CH}_{2} \mathrm{Cl}_{2}$ and the mixture was stirred magnetically at rt. After $4 \mathrm{~h}$, the solution was diluted with 7 $\mathrm{mL}$ of $\mathrm{CH}_{2} \mathrm{Cl}_{2}$ and shaken with water. The organic phase was dried over $\mathrm{MgSO}_{4}$ and the solvent was evaporated. The oily residue was purified by crystallization.

(3-Chloro-3-phenylazetidin-1-yl)(piperidin-1-yl)methanethione (8a). Yield: $90 \mathrm{mg}$ (31\%). Colorless crystals; mp 75-78 ${ }^{\circ} \mathrm{C}(\mathrm{MeOH})$. IR (KBr): 2934m, 1495s, 1464s, 1446s, 1380s, 1348m, 1319m, 1281m, $1252 s, 1222 m, 701 m .{ }^{1} \mathrm{H}-\mathrm{NMR}\left(\mathrm{CDCl}_{3}\right): 1.63-1.65\left(m, 3 \mathrm{CH}_{2}\right), 3.46-3.70\left(m, 2 \mathrm{CH}_{2} \mathrm{~N}\right), 4.74,4.85(A B, J$ $\left.=9.7 \mathrm{~Hz}, \mathrm{CH}_{2} \mathrm{~N}\right), 4.74,4.86\left(A B, J=10.1 \mathrm{~Hz}, \mathrm{CH}_{2} \mathrm{~N}\right), 7.30-7.44(m, 5$ arom. $\mathrm{H}) .{ }^{13} \mathrm{C}-\mathrm{NMR}\left(\mathrm{CDCl}_{3}\right): 24.2$ $\left(\mathrm{CH}_{2}\right), 25.7\left(2 \mathrm{CH}_{2}\right), 50.8\left(2 \mathrm{CH}_{2} \mathrm{~N}\right), 61.4\left(\mathrm{C}_{\mathrm{q}}\right), 70.3\left(2 \mathrm{CH}_{2} \mathrm{~N}\right), 125.5,128.4,128.8$ (5 arom. $\left.\mathrm{CH}\right), 141.4$ (arom. $\mathrm{C}_{\mathrm{q}}$ ), $188.4(\mathrm{C}=\mathrm{S})$. CI-MS: 297 (3), 295 (7, $\left.M^{+}\right), 261$ (17), 260 (18), 259 (100, [M-Cl] ${ }^{+}$). Anal. Calcd for $\mathrm{C}_{15} \mathrm{H}_{19} \mathrm{~N}_{2} \mathrm{ClS}$ : C, 61.10; H, 6.50; N, 9.50; S, 10.88. Found: C, 61.04; H, 6.31; N, 9.43; S, 10.73.

(3-Chloro-3-phenylazetidin-1-yl)(morpholin-4-yl)methanethione (8b). Yield: $70 \mathrm{mg}$ (24\%). Colorless crystals, mp $128-130^{\circ} \mathrm{C}(\mathrm{MeOH})$. IR (KBr): $1474 s, 1443 s, 1431 s, 1348 s, 1308 s, 1278 s, 1231 s, 1114 s .{ }^{1} \mathrm{H}-$ NMR $\left(\mathrm{CDCl}_{3}\right)$ : 4.01-4.08 ( $\left.m, 2 \mathrm{NCH}_{2} \mathrm{CH}_{2} \mathrm{O}\right), 5.06,5.18\left(A B, J=9.8 \mathrm{~Hz}, \mathrm{CH}_{2} \mathrm{~N}\right), 5.07,5.18(A B, J=10.2$ $\left.\mathrm{Hz}, \mathrm{CH}_{2} \mathrm{~N}\right), 7.61-7.72\left(m, 5\right.$ arom. H). ${ }^{13} \mathrm{C}-\mathrm{NMR}\left(\mathrm{CDCl}_{3}\right): 49.8\left(2 \mathrm{CH}_{2} \mathrm{~N}\right), 61.4\left(\mathrm{C}_{\mathrm{q}}\right), 66.3\left(2 \mathrm{CH}_{2} \mathrm{O}\right), 70.3$ $\left(2 \mathrm{CH}_{2} \mathrm{~N}\right), 125.5,128.5,128.8$ (5 arom. CH), 141.2 (arom. $\mathrm{C}_{\mathrm{q}}$ ), 188.9 (C=S). CI-MS: 299 (38), 298 (17, $\left.[M+1]^{+}\right), 297\left(100, M^{+}\right), 263(55), 261\left(40,[M-\mathrm{Cl}]^{+}\right), 217$ (15). Anal. Calcd for $\mathrm{C}_{14} \mathrm{H}_{17} \mathrm{~N}_{2} \mathrm{OClS}$ : C, 56.65; H, 5.77; N, 9.44; S, 10.80. Found: C, 57.06; H, 6.02; N, 9.25; S, 10.18 .

3-Chloro-3-phenylazetidine-1-carbothioic acid N-phenylamide (8c). Yield: $68 \mathrm{mg}$ (22\%). Yellowish crystals; mp $154-158^{\circ} \mathrm{C}$ (hexane/ $\mathrm{CH}_{2} \mathrm{Cl}_{2}$ ). IR (KBr): $1537 s, 1497 m, 1452 s, 1419 m, 1348 m, 696 s .{ }^{1} \mathrm{H}-$ $\operatorname{NMR}\left(\mathrm{CDCl}_{3}\right)$ : 4.58-4.62 ( $\left.m, 2 \mathrm{CH}_{2} \mathrm{~N}\right), 7.10-7.57(m, 10$ arom. $\mathrm{H}) .{ }^{13} \mathrm{C}-\mathrm{NMR}\left(\mathrm{CDCl}_{3}\right): 60.9\left(\mathrm{C}_{\mathrm{q}}\right), 69.0(2$ $\mathrm{CH}_{2} \mathrm{~N}$ ), 125.2, 125.9, 126.7, 129.0, 129.3129 .5 (10 arom. CH), 138.3 (arom. $C_{\mathrm{q}}$ ), 141.4 (arom. CN), 181.7 (C=S). CI-MS: 305 (38), $304\left(21,[M+1]^{+}\right), 303$ (100, $\left.M^{+}\right), 269$ (10), 267 (13), 266 (10). Anal. Calcd for $\mathrm{C}_{16} \mathrm{H}_{15} \mathrm{~N}_{2} \mathrm{ClS}$ : C, 63.46; H, 4.99; N, 9.25; S, 10.59. Found: C, 62.57; H, 5.04; N, 9.05; S, 9.89. 
Reaction of 7 with methanol. General procedure. $1 \mathrm{Mmol}$ of crude 7 was crystallized from $\mathrm{MeOH}$ leading to the substitution product $(\mathbf{9})$.

3-Chloro-3-phenylazetidine-1-carbothioic acid O-methyl ester (9a). Yield: $175 \mathrm{mg}$ (71\%). Colorless crystals; mp $94-96^{\circ} \mathrm{C}$. IR (KBr): $1528 v s, 1492 s, 1448 m, 1432 m, 1279 s, 1268 m, 1234 v s, 1147 m, 696 m$. ${ }^{1} \mathrm{H}-\mathrm{NMR}\left(\mathrm{CDCl}_{3}\right): 4.00(\mathrm{~s}, \mathrm{MeO}), 4.60-4.85\left(m, 2 \mathrm{CH}_{2} \mathrm{~N}\right), 7.25-7.50(m, 5$ arom. $\mathrm{H}) .{ }^{13} \mathrm{C}-\mathrm{NMR}\left(\mathrm{CDCl}_{3}\right)$ : $58.0(\mathrm{MeO}), 61.0\left(\mathrm{C}_{\mathrm{q}}\right), 67.3,68.7\left(2 \mathrm{CH}_{2} \mathrm{~N}\right), 126.0,129.1,129.3$ (5 arom. $\left.\mathrm{CH}\right), 141.5$ (arom. $\left.\mathrm{C}_{\mathrm{q}}\right), 189.4$ $(\mathrm{C}=\mathrm{S})$. CI-MS: $244(37), 243\left(16,[M+1]^{+}\right), 242\left(100, M^{+}\right)$. Anal. Calcd for $\mathrm{C}_{11} \mathrm{H}_{12} \mathrm{NOClS}$ : C, 54.65; H, 5.00; Cl, 14.67; S, 13.27. Found: C, 52.93; H, 4.89; Cl, 14.22; S, 13.00.

3-Chloro-2,2-dimethyl-3-phenylazetidine-1-carbothioic acid O-methyl ester (9b). Yield: $162 \mathrm{mg}$ (59\%). Yellowish crystals; mp 106-109 ${ }^{\circ} \mathrm{C}$. IR (KBr): $1489 v s, 1456 m, 1440 s, 1265 s, 1252 s, 1221 m, 1139 s, 738 s$, 693s. ${ }^{1} \mathrm{H}-\mathrm{NMR}\left(\mathrm{CDCl}_{3}\right)$ : major rotamer: 1.08, $1.83(2 s, 2 \mathrm{Me}), 4.04(s, \mathrm{MeO}), 4.43,4.98(A B, J=12.0 \mathrm{~Hz}$, $\left.\mathrm{CH}_{2} \mathrm{~N}\right)$, 7.29-7.31 (m, 5 arom. H); minor rotamer: 1.32, $2.03(2 s, 2 \mathrm{Me}), 3.97(s, \mathrm{MeO}), 4.43,4.98(A B, J$ $\left.=12.0 \mathrm{~Hz}, 2 \mathrm{CH}_{2} \mathrm{~N}\right), 7.29-7.31(\mathrm{~m}, 5$ arom. $\mathrm{H}) .{ }^{13} \mathrm{C}-\mathrm{NMR}\left(\mathrm{CDCl}_{3}\right)$ : major rotamer: 24.5, $25.1(2 \mathrm{Me})$, $57.4(\mathrm{MeO}), 61.9\left(2 \mathrm{CH}_{2} \mathrm{~N}\right), 71.3\left(\mathrm{Me}_{2} C\right), 77.4\left(\mathrm{C}_{\mathrm{q}}\right), 127.2,128.9$ (5 arom. $\left.\mathrm{CH}\right), 139.3$ (arom. $\left.\mathrm{C}_{\mathrm{q}}\right), 189.7$ $(\mathrm{C}=\mathrm{S})$; minor rotamer: 22.7, $23.8(2 \mathrm{Me}), 56.3(\mathrm{MeO}), 61.9\left(2 \mathrm{CH}_{2} \mathrm{~N}\right), 71.3\left(\mathrm{Me}_{2} \mathrm{C}\right), 77.4\left(\mathrm{C}_{\mathrm{q}}\right), 127.2$, 128.9 (5 arom. CH), 139.3 (arom. $\mathrm{C}_{\mathrm{q}}$ ), $189.7(\mathrm{C}=\mathrm{S})$. CI-MS: $272(36), 271\left(17,[M+1]^{+}\right), 270\left(100, M^{+}\right)$, 131 (13). Anal. Calcd for $\mathrm{C}_{13} \mathrm{H}_{16}$ NOClS: C, 57.88; H, 5.98; N, 5.19; S, 11.89. Found: C, 57.67; H, 5.96; $\mathrm{N}, 5.08 ; \mathrm{S}, 11.91$.

3-Chloro-2,2-dimethyl-3-(4-fluorophenyl)azetidine-1-carbothioic acid O-methyl ester (9c). Yield: $165 \mathrm{mg}$ (57\%). Yellowish crystals; mp $116-118^{\circ} \mathrm{C}$. IR (KBr): 1496s, 1266m, 1253m, 1234m. ${ }^{1} \mathrm{H}-\mathrm{NMR}\left(\mathrm{CDCl}_{3}\right)$ : major rotamer: 1.08, $1.81(2 s, 2 \mathrm{Me}), 4.03(s, \mathrm{MeO}), 4.43,4.93\left(A B, J=12.0 \mathrm{~Hz}, 2 \mathrm{CH}_{2} \mathrm{~N}\right), 6.93-7.47(m$, 5 arom. $\mathrm{H})$; minor rotamer: 1.30, $2.03(2 s, 2 \mathrm{Me}), 3.97(s, \mathrm{MeO}), 4.43,4.93\left(A B, J=12.0 \mathrm{~Hz}, 2 \mathrm{CH}_{2} \mathrm{~N}\right)$, 6.93-7.47 (m, 5 arom. H). ${ }^{13} \mathrm{C}-\mathrm{NMR}\left(\mathrm{CDCl}_{3}\right)$ : major rotamer: 24.7, $24.9(2 \mathrm{Me}), 57.4(\mathrm{MeO}), 61.8(2$ $\left.\mathrm{CH}_{2} \mathrm{~N}\right), 70.4\left(\mathrm{Me}_{2} C\right), 77.2\left(\mathrm{C}_{\mathrm{q}}\right), 115.6\left(d,{ }^{2} J_{\mathrm{C}, \mathrm{F}}=21.7 \mathrm{~Hz}, 2\right.$ arom. $\left.\mathrm{CH}\right), 128.8\left(d,{ }^{3} J_{\mathrm{C}, \mathrm{F}}=8.2 \mathrm{~Hz}, 2\right.$ arom. $\mathrm{CH}), 134.9\left(d,{ }^{4} J_{\mathrm{C}, \mathrm{F}}=3.4 \mathrm{~Hz}\right.$, arom. $\left.\mathrm{C}_{\mathrm{q}}\right), 162.4\left(d,{ }^{1} J_{\mathrm{C}, \mathrm{F}}=249.0 \mathrm{~Hz}\right.$, arom. $\left.\mathrm{C}_{\mathrm{q}} \mathrm{F}\right), 189.1(\mathrm{C}=\mathrm{S})$; minor rotamer: 22.7, $23.7(2 \mathrm{Me}), 56.2(\mathrm{MeO}), 61.8\left(2 \mathrm{CH}_{2} \mathrm{~N}\right), 71.9\left(\mathrm{Me}_{2} C\right), 78.3\left(\mathrm{C}_{\mathrm{q}}\right), 115.6\left(d,{ }^{2} J_{\mathrm{C}, \mathrm{F}}=21.7 \mathrm{~Hz}\right.$, 2 arom. $\mathrm{CH}), 128.8\left(d,{ }^{3} J_{\mathrm{C}, \mathrm{F}}=8.2 \mathrm{~Hz}, 2\right.$ arom. CH), $134.9\left(d,{ }^{4} J_{\mathrm{C}, \mathrm{F}}=3.4 \mathrm{~Hz}\right.$, arom. $\left.\mathrm{C}_{\mathrm{q}}\right), 162.4\left(d,{ }^{1} J_{\mathrm{C}, \mathrm{F}}=\right.$ $249.0 \mathrm{~Hz}$, arom. $\left.\mathrm{C}_{\mathrm{q}} \mathrm{F}\right), 189.1$ (C=S). CI-MS: $290(38), 289\left(15,[M+1]^{+}\right), 288\left(100, M^{+}\right), 254(10), 252(9$, $\left.[M-\mathrm{Cl}]^{+}\right)$. Anal. Calcd for $\mathrm{C}_{13} \mathrm{H}_{15} \mathrm{NOClFS}$ : C, 54.26; H, 5.98; N, 4.87; S, 11.14. Found: C, 54.48; H, 5.88; $\mathrm{N}, 4.98 ; \mathrm{S}, 11.11$. 
X-Ray Crystal-Structure Determination of 6a (see Table 1 and Figure 1). ${ }^{28}$ All measurements were performed on a Nonius KappaCCD area-detector diffractometer ${ }^{29}$ using graphite-monochromated $\mathrm{MoK}_{a}$ radiation $(\lambda 0.71073 \AA)$ and with an Oxford Cryosystems Cryostream 700 cooler. The data collection and

Table 1. Crystallographic Data of Compound (6a)

Crystallized from

Empirical formula

Formula weight $\left[\mathrm{g} \mathrm{mol}^{-1}\right]$

Crystal color, habit

Crystal dimensions [mm]

Temperature $[\mathrm{K}]$

Crystal system

Space group

Z

Reflections for cell determination

$2 \theta$ range for cell determination $\left[{ }^{\circ}\right]$

Unit cell parameters

$$
\begin{aligned}
& a[\AA] \\
& b[\AA] \\
& c[\AA] \\
& \beta\left[^{\circ}\right] \\
& V\left[\AA^{3}\right]
\end{aligned}
$$

$D_{x}\left[\mathrm{~g} \mathrm{~cm}^{-3}\right]$

$\mu\left(\mathrm{MoK}_{\alpha}\right)\left[\mathrm{mm}^{-1}\right]$

Scan type

$2 \theta(\max )\left[^{\circ}\right]$

Transmission factors (min; max)

Total reflections measured

Symmetry independent reflections

Reflections with $I>2 \sigma(I)$

Reflections used in refinement

Parameters refined

Final $R(F)[I>2 \sigma(I)$ reflections $]$

$$
w R\left(F^{2}\right) \text { (all data) }
$$

\section{$\mathrm{MeOH} / \mathrm{CH}_{2} \mathrm{Cl}_{2}$}

$\mathrm{C}_{16} \mathrm{H}_{14} \mathrm{O}_{2} \mathrm{~S}_{4}$

319.87

colorless, prism

$0.12 \times 0.30 \times 0.32$

273(1)

monoclinic

$P 21 / c$

4

20580

4-55

9.2577(2)

18.3804(4)

8.9874(2)

94.382(1)

1524.83(6)

1.393

0.512

$\phi$ and $\omega$

55

$0.836 ; 0.942$

32689

3489

2776

3488

181

0.0395

0.1046

Weights: $w=\left[\sigma^{2}\left(F_{\mathrm{o}}{ }^{2}\right)+(0.0493 P)^{2}+0.7442 P\right]^{-1}$ where $P=\left(F_{\mathrm{o}}{ }^{2}+2 F_{\mathrm{c}}{ }^{2}\right) / 3$

Goodness of fit

Final $\Delta_{\max } / \sigma$

0.001

$\Delta \rho(\max ; \min )\left[\mathrm{e} \AA^{-3}\right]$

$0.28 ;-0.51$ 
refinement parameters are given in Table 1, and a view of the molecule is shown in Figure 1. Data reduction for was performed with HKL Denzo and Scalepack. ${ }^{30}$ The intensities were corrected for Lorentz and polarization effects, and an absorption correction based on the multi-scan method ${ }^{31}$ was applied. The structure was solved by direct methods using SIR92, ${ }^{32}$ which revealed the positions of all non-H-atoms. The non-H-atoms were refined anisotropically. All of the H-atoms were placed in geometrically calculated positions and refined using a riding model where each atom was assigned a fixed isotropic displacement parameter with a value equal to $1.2 U_{\text {eq }}$ of its parent $\mathrm{C}$-atom. The refinement of the structure was carried out on $F^{2}$ using full-matrix least-squares procedures, which minimized the function $\Sigma w\left(F_{\mathrm{o}}{ }^{2}-\right.$ $\left.F_{\mathrm{c}}{ }^{2}\right)^{2}$. A correction for secondary extinction was not applied. One reflection, whose intensity was considered to be an extreme outlier, was omitted from the final refinement. Neutral atom scattering factors for non-H-atoms were taken from ref. ${ }^{33 a}$, and the scattering factors for H-atoms were taken from ref. $^{34}$ Anomalous dispersion effects were included in $F_{\mathrm{c}} ;^{35}$ the values for $f$ and $f^{\prime}$ were those of ref. ${ }^{33 b}$ The values of the mass attenuation coefficients are those of ref. ${ }^{33 \mathrm{c}}$ All calculations were performed using the SHELXL97 program. $^{36}$

\section{ACKNOWLEDGEMENT}

G. M., M. W. and K. U. thank the Polish State Committee for Scientific Research for financial support (Grant KBN No. 4 T09A 046 25); H. H. acknowledges financial support by F. Hoffmann-La Roche AG, Basel. We thank PD Dr. A. Linden, University of Zürich, for the crystal-structure determination.

\section{REFERENCES AND NOTES}

1. $\quad$ Part of the planned Ph. D. thesis of $M . W$., University of Lodz.

2. R. Bartnik and A. P. Marchand, Synlett, 1997, 1029.

3. K. Hayashi, C. Sato, S. Hiki, T. Kumagai, S. Tamai, T. Abe, and Y. Nagao, Tetrahedron Lett., 1999, 40, 3761.

4. Some polycyclic compounds containing 1-azabicyclo[1.1.0]butane fragments were described by Prinzbach and coworkers. ${ }^{5 \mathrm{a}}$ On the other hand, 2-chloro substituted derivatives were postulated as reactive intermediates responsible for the in situ formation of unstable azacyclobutadiene. ${ }^{5 b}$

5. a) B. Trupp, H. Fritz, H. Prinzbach, H. Irngartinger, and U. Reifenstahl, Chem. Ber., 1991, 124, 1777; b) T. Tsuritani, K. Yagi, H. Shinokubo, and K. Oshima, Angew. Chem. Int. Ed., 2003, 42, 5613.

6. a) K. Hayashi, S. Hiki, T. Kumagai, and Y. Nagao, Heterocycles, 2002, 56, 433; b) G. Mloston and H. Heimgartner, Helv. Chim. Acta, 2006, 89, 442.

7. R. Bartnik, S. Lesniak, G. Mloston, and J. Romanski, Pol. J. Chem., 1994, 68, 1347. 
8. A. Jackson, Chimicaoggi/chemistry today, 1994, 33.

9. S. Sharma, Synthesis, 1978, 803.

10. K. Matsumo, T. Nakajima, M. Ichimura, N. A. Giese, J.-C. Yu, N. A. Lokker, J. Ushiki, S. Ide, S. Oda, and Y. Nomoto, J. Med. Chem., 2002, 45, 4513.

11. C. Che, G. Petit, F. Kotzyba-Hilbert, S. Bertrand, D. Bertrand, T. Grütter, and M. Goeldner, Bioorg. Med. Chem. Lett., 2003, 13, 1001.

12. U. Kraatz, in 'Methoden der Organischen Chemie (Houben-Weyl)', Vol. E4, Ed. H. Hagemann, Thieme, Stuttgart, 1983, p. 414.

13. I. El. Sayed, F. M. Abdel-Megeed, S. Yassin, and A. Senning, Sulfur Rep., 1995, 16, 235.

14. K. Kanie, K. Mizuno, M. Kuroboshi, and T. Hiyama, Bull. Chem. Soc. Jpn., 1998, 71, 1973.

15. H. Ried, Liebigs Ann. Chem., 1954, 590, 128.

16. C. Len, D. Postel, G. Ronzo, P. Villa, and C. Goubert, J. Agric. Food Chem., 1997, 45, 3.

17. D. A. Tomalia, J. Heterocycl. Chem., 1966, 3, 384.

18. In the analogous 3-chloro-1-(methoxycarbonyl)-3-phenylazetidine, the two $\mathrm{CH}_{2}$ groups absorb as a broad singlet at $4.63\left({ }^{1} \mathrm{H}\right)$ and as a singlet at $65.8 \mathrm{ppm}\left({ }^{13} \mathrm{C}\right)$, respectively. ${ }^{7}$

19. a) R. C. Neuman, Jr., D. N. Roark, and V. Jonas, J. Am. Chem. Soc., 1967, 89, 3412. b) W. Walter, C. Maerten, and M. Rose, Liebigs Ann. Chem., 1966, 691, 25. c) A. Mannschreck, Angew. Chem., Int. Ed. Engl., 1965, 4, 985. d) A. Lowenstein, A. Melera, P. Rigny, and W. Walter, J. Phys. Chem., 1964, 68, 1598.

20. a) J. R. Grunwell, J. Org. Chem., 1970, 35, 1500. b) Z.-C. Chen. Y.-Y. Jin, and P. J. Stang, J. Org. Chem., 1987, 52, 4117.

21. a) K. Takagi, H. Takachi, and K. Sasaki, J. Org. Chem., 1995, 60, 6552. b) M. Koketsu, T. Otsuka, and H. Ishihara, Phosphorus, Sulfur, Silicon, 2004, 179, 443.

22. C. K. Johnson, ORTEP II, Report ORNL-5138, Oak Ridge National Laboratory, Oak Ridge, Tennessee, 1976.

23. G. J. Martin, J. P. Gouesnard, J. Dorie, C. Rabiller, and M. L. Martin, J. Amer. Chem. Soc., 1977, 99, 1381.

24. a) F. A. L. Anet and M. Ghiaci, J. Am. Chem. Soc., 1979, 101, 6857. b) R. H. Sullivan and E. Price, Org. Magn. Res., 1975, 7, 143.

25. A. G. Hortmann and D. A. Robertson, J. Am. Chem. Soc., 1972, 94, 2758.

26. I. El-Sayed, M. F. Abdel-Megeed, S. M. Yassin, and A. Senning, Phosphorus, Sulfur and Silicon, 1994, 86, 239.

27. J. Goerdeler and H. Hohage, Chem. Ber., 1973, 106, 1487. 
28. CCDC-611938 contains the supplementary crystallographic data for compound (6a). These data can be obtained free of charge via www.ccdc.cam.ac.uk/data_request/cif.

29. R. Hooft, KappaCCD Collect Software, Nonius BV, Delft, The Netherlands, 1999.

30. Z. Otwinowski and W. Minor, in Methods in Enzymology, Vol. 276, Macromolecular Crystallography, Part A, ed. by C. W. Carter Jr. and R. M. Sweet, Academic Press, New York, 1997, p. 307.

31. R. H. Blessing, Acta Crystallogr., Sect. A, 1995, 51, 33.

32. A. Altomare, G. Cascarano, C. Giacovazzo, A. Guagliardi, M. C. Burla, G. Polidori, and M. Camalli, SIR92, J. Appl. Crystallogr., 1994, 27, 435.

33. a) E. N. Maslen, A. G. Fox, and M. A. O'Keefe, in International Tables for Crystallography, ed. by A. J. C. Wilson, Kluwer Academic Publishers, Dordrecht, 1992, Vol. C, Table 6.1.1.1, p. 477. b) D. C. Creagh and W. J. McAuley, ibid. Table 4.2.6.8, p. 219. c) D. C. Creagh and J. H. Hubbell, ibid. Table 4.2.4.3, p. 200.

34. R. F. Stewart, E. R. Davidson, and W. T. Simpson, J. Chem. Phys., 1965, 42, 3175.

35. J. A. Ibers and W. C. Hamilton, Acta Crystallogr., 1964, 17, 781.

36. G. M. Sheldrick, SHELXL97, Program for the Refinement of Crystal Structures, University of Göttingen, Germany, 1997. 